\title{
1 Evolutionary flexibility in routes to mat formation by Pseudomonas
}

2

3 Anuradha Mukherjee ${ }^{1,2}$ and Jenna Gallie ${ }^{1^{*}}$

4

$5 \quad{ }^{1}$ Max Planck Institute for Evolutionary Biology, Department of Evolutionary Theory, 24306 Plön, Germany

$6{ }^{2}$ Current address: Institute of Experimental Medicine, Christian-Albrechts University (UKSH Campus), Kiel,

7 Germany

$8 *$ corresponding author

ABSTRACT

Many bacteria form mats at the air-liquid interface of static microcosms. These structures typically involve the secretion of exopolysaccharide(s), the production of which is often controlled by the secondary messenger c-di-GMP. Mechanisms of mat formation have been particularly well characterized in Pseudomonas fluorescens SBW25; mutations that lead to an increase in c-di-GMP production by diguanylate cyclases (WspR, AwsR, or MwsR) result in the secretion of cellulose, and mat formation. Here, we characterize and compare mat formation in two close relatives of SBW25: Pseudomonas simiae PICF7 and Pseudomonas fluorescens A506. We find that PICF7 - the strain more closely related to SBW25 - can form mats through mutations affecting the activity of the same three diguanylate cyclases as SBW25. However, instead of cellulose, these mutations activate the production of the Pel exopolysaccharide. We also provide evidence for at least two further - as yet uncharacterized - routes to PICF7 mat formation. P. fluorescens A506, while retaining the same mutational routes to mat formation as SBW25 and PICF7, forms mats by a semi-heritable mechanism that likely culminates in Pga and/or Psl production. Overall, our results demonstrate a high level of evolutionary flexibility in the molecular and structural routes to mat formation, even among close relatives.

KEYWORDS Biofilms, polysaccharides, cellulose, Pel, Pseudomonas fluorescens, Pseudomonas simiae

\section{INTRODUCTION}

Many bacteria can form structures on solid surfaces (biofilms) or at the air-liquid interface of static microcosms (mats or pellicles). The formation of these structures is dependent on the production and excretion of exopolysaccharides (EPSs) by the bacterial cells. EPS production can afford bacterial cells protection against stresses such as desiccation or starvation (Davey and O'Toole, 2000), and biofilms have been shown to increase pathogen persistence in the face of the host immune system and treatment regimens (Costerton, 1999). Given the prevalence of biofilms and mats, the evolutionary and molecular mechanisms behind their production are of interest. Here, we characterize mat 
production in two biocontrol agents, Pseudomonas simiae PICF7 and Pseudomonas fluorescens A506, and contrast them with previously well studied routes in Pseudomonas fluorescens SBW25.

The process of mat and biofilm formation often involves the concerted production of multiple structural components, and hence is under complex control. The biosynthesis of many structural components is regulated by the secondary messenger bis $\left(3^{\prime}-5^{\prime}\right)$-cyclic dimeric guanosine monophosphate (c-di-GMP) (Ross et al., 1987; Römling et al., 2013; Liang, 2015). C-di-GMP is produced from GTP by enzymes called diguanylate cyclases (DGCs) with active GGDEF domains (Tal et al., 1998). Conversely, c-di-GMP is broken down by phosphodiesterases (PDEs), the activity of which is dependent on conserved EAL or HD-GYP domains (Schmidt et al., 2005). Most bacteria encode multiple DGCs and PDEs, the combined activities of which determine local and global intracellular c-di-GMP levels.

DGC and PDE activity is regulated by a range of stimuli, using various signal transduction pathways. A common mechanism of signal transduction in bacteria is the two-component regulatory system (Nixon et al., 1986; Stock et al., 2000). In such systems, a signal activates the first component (a histidine kinase), which passes the signal by a phosphotransfer event to the second component (a response regulator). Response regulators include several known examples of c-di-GMP metabolizing enzymes, including WspR of $P$. fluorescens (Bantinaki et al., 2007; McDonald et al., 2009) and PleD of Caulobacter crescentus (Paul, 2004; Chan et al., 2004). In both cases, activation of a twocomponent signal transduction pathway results in c-di-GMP production and, ultimately, a switch from motility to a non-motile, sessile lifestyle.

The downstream targets of c-di-GMP are notably varied; known examples fall into four categories: (i) PilZ domains (Amikam and Galperin, 2006; Ryjenkov et al., 2006; Lee et al., 2007), (ii) degenerate, enzymatically inactive GGDEF/EAL domains (Duerig et al., 2009; Colvin et al., 2012; Li et al., 2012), (iii) other, distinct c-di-GMP binding domains in transcriptional regulators (Hickman and Harwood, 2008; Baraquet et al., 2012), and (iv) mRNA riboswitches (Sudarsan et al., 2008; Chen et al., 2011). Overall, c-di-GMP regulates a plethora of targets, at the levels of transcription, translation, and enzymatic activity. Many EPSs are downstream targets of c-di-GMP, including cellulosic polymers in Escherichia coli, Acetobacter xylinum, and P. fluorescens (Ross et al., 1987; Goymer et al., 2006; Richter et al., 2020), Pga in E. coli and P. fluorescens (Boehm et al., 2009; Steiner et al., 2012; Lind et al., 2017), and Pel in Pseudomonas aeruginosa and Bacillus cereus (Hickman and Harwood, 2008; Whitfield et al., 2020).

$P$. fluorescens SBW25 is a model evolutionary system in which the mechanisms of mat formation have been particularly well characterized. SBW25 readily forms mats, or pellicles, at the air-liquid interface of static microcosms (Rainey and Travisano, 1998). The major structural component of these mats is an acetylated cellulosic polymer (Spiers et al., 2003) or, when cellulose is unavailable, Pga (Lind et al., 2017). Both EPSs are produced in response to high levels of c-di-GMP (Goymer et al., 2006; Malone et al., 2007; Lind et al., 2017). In the case of cellulose, c-di-GMP is 
thought to target the biosynthetic machinery (Wss) by binding to the PilZ domain of WssB. The mechanism by which c-di-GMP activates SBW25 Pga has not been elucidated, but may resemble the E. coli mechanism of c-di-GMP binding to, and activating, Pga pathway components (Boehm et al., 2009; Steiner et al., 2012). In laboratory SBW25 populations, elevated c-di-GMP levels are caused by mutations affecting the activity of one of three DGCs/PDEs: WspR (DGC), AwsR (DGC), MwsR (DGC/PDE) (Bantinaki et al., 2007; McDonald et al., 2009; Gallie et al., 2019; Lind et al., 2019). The environmental signals that activate these pathways in the absence of mutation remain unknown.

In this work, we characterize and compare mat formation in two relatives of $P$. fluorescens SBW25: P. simiae PICF7 and P. fluorescens A506. We find that, despite their relatively close evolutionary relationship, each strain produces mats by distinct molecular and structural mechanisms.

\section{RESULTS}

Evolutionary relationships: PICF7 is more closely related to SBW25 than is A506

In this study we concentrate on three Pseudomonas strains: (i) $P$. fluorescens SBW25, isolated from a sugar beet leaf in the United Kingdom, (ii) P. simiae PICF7 (formerly P. fluorescens PICF7), isolated from the roots of an olive plant in Spain, and (iii) P. fluorescens A506, isolated from a pear plant in the USA (Table S1). All three strains have a symbiotic relationship with plants, and PICF7 and A506 are biocontrol agents for Verticillum wilt of olive (Mercado-Blanco et al., 2004) and fire blight of pear and apple (Stockwell et al., 2010), respectively. Complete genome sequences are available (Silby et al., 2009; Loper et al., 2012; Martínez-García et al., 2015), and A506 also contains a sequenced, $\sim 57 \mathrm{~kb}$ conjugative plasmid (pA506; Stockwell et al., 2013). Phylogenetic analyses indicate that $P$. fluorescens SBW25 and P. simiae PICF7 more recently shared a common ancestor with each other than with P. fluorescens A506 (Figure 1).

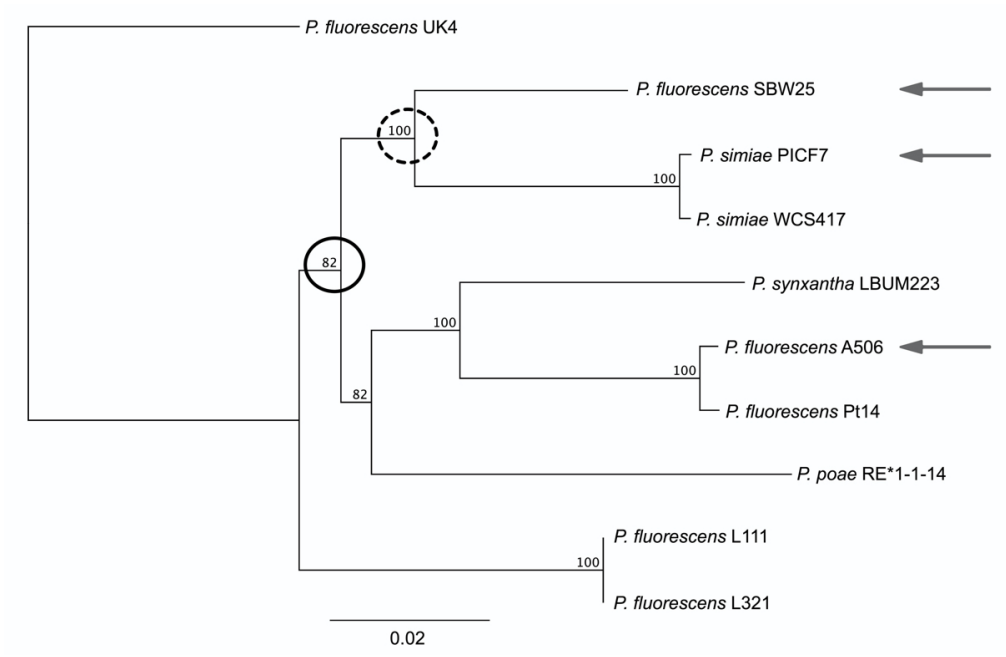

Figure 1. Phylogenetic tree highlighting the evolutionary relationships between $P$. fluorescens SBW25, $P$. simiae PICF7, and P. fluorescens A506. The three strains of interest are indicated by grey arrows. The last common ancestor of SBW25 and PICF7 is indicated by the dotted circle, and the last common ancestor of all three is indicated by a solid circle. The support shown next to the branches is based on 100 bootstrap replicates. 
SBW25, PICF7 and A506 encode the biosynthetic genes required to synthesize distinct sets of EPSs; each strain carries genes for Pga, Psl, and alginate biosynthesis, while SBW25 and PICF7 encode genes for the biosynthesis of additional polymers (cellulose and colanic acid in SBW25, Pel in PICF7) (Table 1; Table S2). In addition, all three strains carry homologues of the $w s p$, aws, and $m w s R$ loci, each of which is predicted to contain at least one c-di-GMP metabolizing domain (DGC/PDE; Table S2), Hence, SBW25, PICF7, and A506 each has the genetic capacity to over-produce c-diGMP, with a strain-specific set of possible downstream (EPS) targets.

\begin{tabular}{l|l|c|c|c}
\hline Locus & Function & SBW25 & PICF7 & A506 \\
\hline$w s s$ & Cellulose biosynthesis & $\checkmark$ & $\mathbf{x}$ & x \\
$w c a$ & Colanic acid capsule biosynthesis & $\checkmark$ & $\mathbf{x}$ & $\mathbf{x}$ \\
$p e l$ & Pel biosynthesis & $\checkmark$ & $\checkmark$ & $\mathbf{x}$ \\
$p g a$ & Pga biosynthesis & $\checkmark$ & $\checkmark$ & $\checkmark$ \\
$p s l$ & Psl biosynthesis & $\checkmark$ & $\checkmark$ & $\checkmark$ \\
$a l g$ & Alginate biosynthesis & $\checkmark$ & $\checkmark$ & $\checkmark$ \\
$w s p$ & Regulation of EPS production & $\checkmark$ & $\checkmark$ & $\checkmark$ \\
$a w s$ & Regulation of EPS production & $\checkmark$ & $\checkmark$ & $\checkmark$ \\
$m w s R$ & Regulation of EPS production & & $\checkmark$ \\
\hline
\end{tabular}

Table 1. A (non-exhaustive) list of genetic loci involved in EPS production in SBW25, PICF7, and A506.

Further details of selected loci are provided in Table S2. Synonyms include awsXRO/tpbB/yfiBNR; mwsR/morA.

PICF7 and A506 form emergent mats and diverse colony morphotypes in static microcosms

Mat formation by PICF7 and A506 was investigated relative to SBW25. Ten single (smooth, wildtype) colonies from each of the three strains were inoculated into separate microcosms, and incubated statically. By 72 hours, mats had emerged at the air-liquid interface of all microcosms. The mats formed from PICF7 were very similar in appearance to those formed from SBW25, while A506 mats were noticeably thinner, less defined, and usually less fluorescent (Figure 2A). Plating of cells from the 72-hour microcosms revealed the development of considerable colony diversity (Figure 2B). As expected from previous studies, SBW25 gave rise to three distinct morphotypes (Smooth, Wrinkly Spreader, Fuzzy Spreader) (Rainey and Travisano, 1998). Five distinct morphotype classes were observed arising from the majority of PICF7 microcosms (Smooth, Large Smooth, Wrinkly Spreader, Small Disc, Large Disc), while A506 microcosms typically gave rise to two morphotype classes (Smooth, Web). Notably, the PICF7 wrinkly spreaders (WS) closely resembled the mat forming WS morphotype of SBW25, and were seen arising from all ten microcosms. No wrinkly spreader colonies were observed arising from any of the A506 microcosms.

While PICF7 and A506 form mats in 72-h microcosms, the mats typically require between 48 and 72 hours to fully emerge. To test which, if any, of the emergent colony morphotypes gained the 
ability to form stable mats more quickly, examples of each morphotype were purified and inoculated into fresh microcosms. After 24 hours, five of the ten morphotypes formed a stable mat: SBW25 wrinkly spreader (SBW25-WS), PICF7 wrinkly spreader (PICF7-WS), PICF7 large smooth (PICF7LSm), PICF7 small disc (PICF7-SD), and A506 web (A506-Web) (Figure 2C, Figure S1).

A

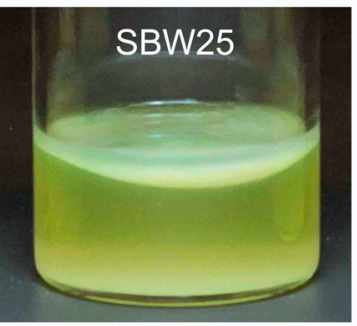

B
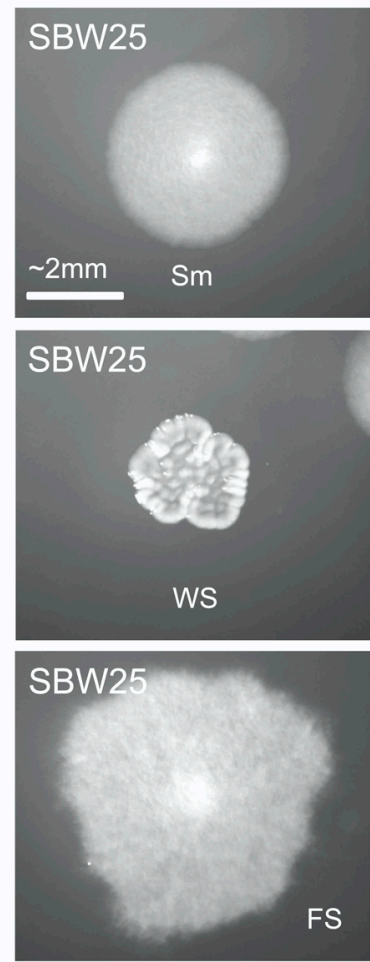

C

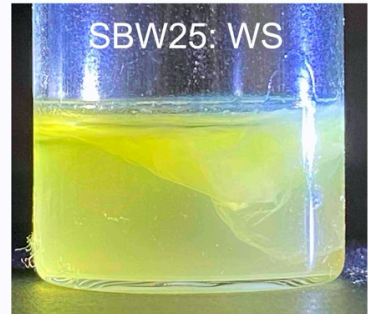

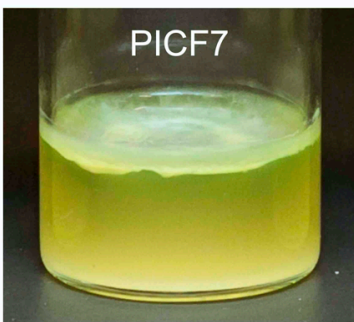
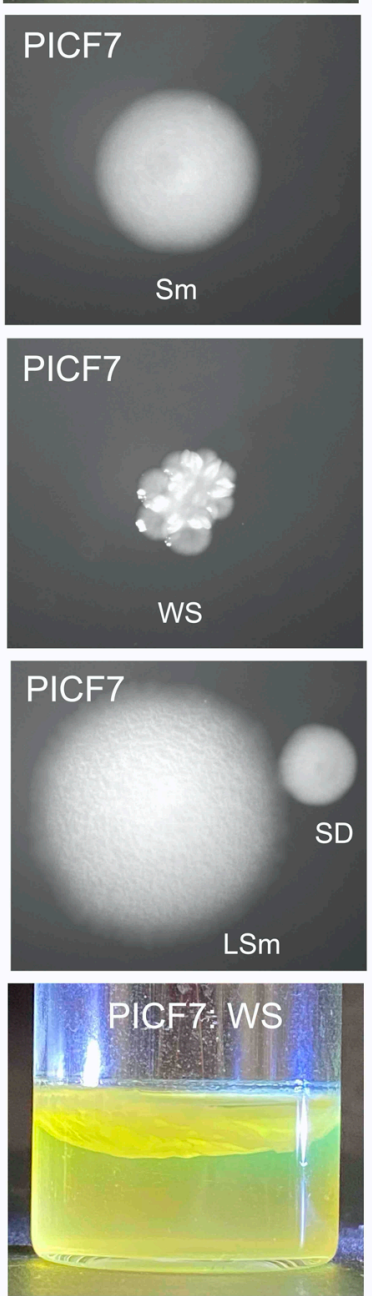
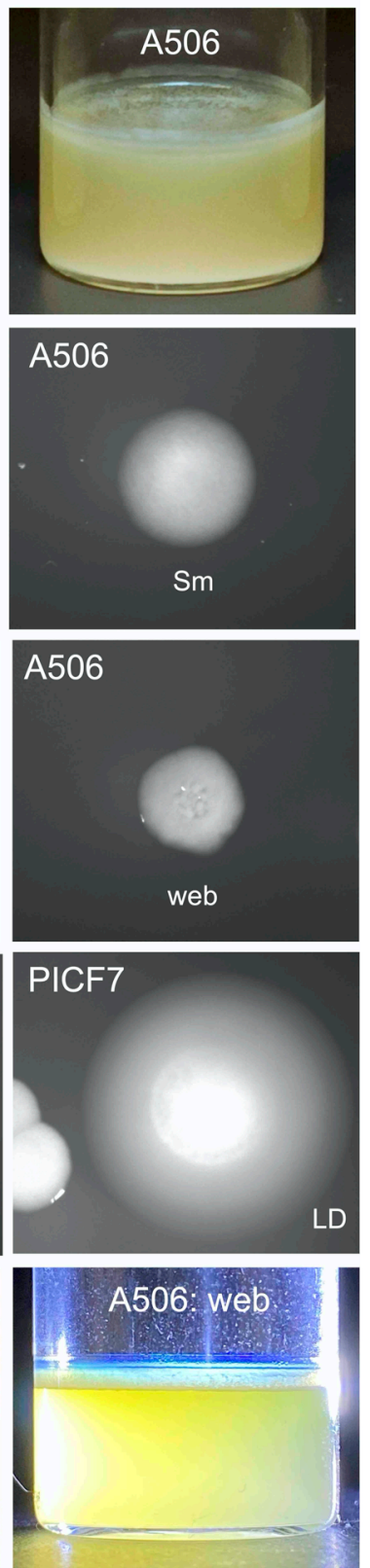

Figure 2. Mat formation by, and emergent colony diversity in, PICF7 and A506 relative to SBW25. (A)

Representative 72-hour microcosms founded by SBW25, PICF7, and A506. All three strains develop mats at the air-liquid interface within 72 hours. (B) Dilution plating from microcosms shows ten morphotypes: three in SBW25 (Smooth (Sm), Wrinkly Spreader (WS), Fuzzy Spreader (FS)), five in PICF7 (Smooth (Sm), Wrinkly Spreader (WS), Large Smooth (LSm), Large Disc (LD), and Small Disc (SD)), and two in A506 (Smooth (Sm), and Web). (C) SBW25-WS, PICF7-WS, and A506-Web give rise to mats at the air-liquid interface within 24 hours. See Figure S1 for 24-h microcosms from other morphotypes. Brightness and exposure of some images were altered in Preview. 

microcosms, and that mat formation is accompanied by the emergence of a range of colony morphotypes. In PICF7, at least three morphotypes may contribute to the 72-h mat (WS, LSm, SD), while the A506 mat is likely underpinned by the web morphotype. In the remainder of this work, we concentrate on characterizing the molecular mechanisms underpinning mat formation in the emergent PICF7-WS and A506-Web morphotypes.

PICF7 wrinkly spreaders carry mutations in wsp, aws, or mwsR

9 The mats and WS colonies observed from PICF7 static microcosms strongly resemble their SBW25 counterparts. That is, they have the emergent property of forming a stable mat within 24 hours, and the wrinkly phenotype is heritably stable (i.e., when PICF7-WS colonies are sub-streaked on agar, they give rise to exclusively WS colonies). In order to determine whether the same mutational pathways underpin the WS phenotype in PICF7 as SBW25, ten WSs were isolated from independent, 72-h microcosms founded by PICF7 (giving PICF7-WS1 to 10) and SBW25 (giving SBW25-WS1 to 10). Whole genome sequencing of these twenty WS genotypes revealed a mutation in $w s p$, aws, or $m w s R$ in every case (Table 2; Text S1). Six PICF7-WS types carried mutations in the wsp locus (versus four SBW25-WSs), three in the aws locus (versus two SBW25-WSs), and one in $m w s R$ (versus four SBW25-WSs). Each mutation is predicted to result in c-di-GMP over-production by WspR, AwsR, or MwsR, leading to the activation of EPS biosynthesis and mat formation (Text S1).

\begin{tabular}{c|c|c|c|c|c}
\hline Gene & Locus tag & $\begin{array}{c}\text { Isolate } \\
\text { number }\end{array}$ & $\begin{array}{c}\text { Nucleotide } \\
\text { change }\end{array}$ & Protein change & Ref $^{\dagger}$ \\
\hline
\end{tabular}

\begin{tabular}{|c|c|c|c|c|c|}
\hline \multicolumn{6}{|c|}{ PICF7-WS } \\
\hline$w s p A$ & PFLUOLIPICF7_RS02250 & 1 & $\mathrm{c} 161 \mathrm{t}$ & S54L & \\
\hline$w s p F$ & PFLUOLIPICF7_RS02225 & 2 & $\mathrm{c} 250 \mathrm{t}$ & R82(STOP) & \\
\hline$w s p F$ & PFLUOLIPICF7_RS02225 & 3 & c747g & I248(STOP) & \\
\hline$w s p F$ & PFLUOLIPICF7_RS02225 & 8 & a880c & T294P & \\
\hline$w s p F$ & PFLUOLIPICF7_RS02225 & 9 & $\Delta 949-951$ & $\Delta \mathrm{A} 317$ & \\
\hline$w s p F$ & PFLUOLIPICF7_RS02225 & 10 & $\mathrm{c} 898 \mathrm{t}$ & Q300(STOP) & \\
\hline aws $X$ & PFLUOLIPICF7_RS12635 & 4,5 & $\Delta 232-264$ & $\begin{array}{l}\Delta 78-88 \\
(\Delta Y T D D L I K G T T Q)\end{array}$ & $2-6$ \\
\hline awsO & PFLUOLIPICF7_RS12645 & 6 & $\mathrm{t} 127 \mathrm{a}$ & F43I & \\
\hline$m w s R$ & PFLUOLIPICF7_RS12125 & 7 & g2976t & M992I & \\
\hline \multicolumn{6}{|c|}{$S B W 25-W S$} \\
\hline$w s p A$ & PFLU1219 & 9 & $\mathrm{c} 1313 \mathrm{t}$ & S438F & \\
\hline$w s p E$ & PFLU1223 & 10 & g1912t & D638Y & 6 \\
\hline$w s p F$ & PFLU1224 & 4 & $\Delta 151-165$ & $\Delta 51-55(\Delta \mathrm{LMDLI})$ & 1 \\
\hline$w s p F$ & PFLU1224 & 8 & $\Delta 677-826$ & $\Delta 226-275(\Delta 50$ residues $)$ & 5 \\
\hline
\end{tabular}




\begin{tabular}{|c|c|c|c|c|c|}
\hline$a w s R$ & PFLU5210 & 6 & a79c & T27P & \\
\hline awsR & PFLU5210 & 7 & g574a & A192T & \\
\hline$m w s R$ & PFLU5329 & 1 & g3244a & E1082K & \\
\hline$m w s R$ & PFLU5329 & 2 & a2476t & S826C & \\
\hline$m w s R$ & PFLU5329 & 3 & $\mathrm{t} 2183 \mathrm{c}$ & V728A & \\
\hline$m w s R$ & PFLU5329 & 5 & g3095t & R1032L & 2 \\
\hline
\end{tabular}

Table 2. Details of mutations identified in ten WS strains isolated from each of PICF7 and SBW25.

2 tReference is provided if a change in the same amino acid(s) has previously been reported to cause the WS

3 phenotype in P. fluorescens SBW25. 1=Bantinaki et al., 2007, 2=Gallie et al., 2015, 3=Gallie et al., 2019,

4 4=Lind et al., 2017, 5=Lind et al., 2019, 6=McDonald et al., 2009. See Text S1 for further molecular details.

6 The Pel polymer contributes to the structure of the PICF7 wrinkly spreader phenotype

7 Despite the phenotypic and genotypic similarities between PICF7-WS and SBW25-WS, the mats

8 formed by each must differ on a structural level because PICF7 lacks homologues of the cellulose-

9 biosynthetic wss genes that are activated by wsp, aws, or $m w s R$ mutations in SBW25-WS. However,

10 PICF7 carries several other polymer biosynthetic loci that could conceivably be activated by $w s p$, aws, or $m w s R$ mutations (see Table 1). To investigate the structural basis of the PICF7-WS phenotype, a suppressor analysis was performed on PICF7-WS3, -WS4, -WS7, and -WS8 (carrying mutations in $w s p F, a w s X, m w s R$, and $w s p F$, respectively). Each genotype was subjected to random transposon mutagenesis, and transposon mutants that had reverted to the smooth colony phenotype, and lost the ability to form 24-h mats in static microcosms, were obtained. The genomic location of the transposon was determined in these mutants. Overall, approximately 15,600 transposon mutants were screened, and the insertion site determined in 100 suppressor mutants (Table S4).

Multiple independent transposon insertions were obtained in two types of loci. Firstly, 29 independent insertions were obtained in the $w s p$, aws, or $m w s R$ loci. Presumably, these insertions directly counteract the WS mutation in each background, lowering c-di-GMP levels and reversing the WS phenotype. Secondly, 14 independent insertions were obtained in the seven-gene EPS biosynthetic locus, pelA-G (PFLUOLIPICF7_RS06785-PFLUOLIPICF7_RS06755; Figure 3A). It is probable that this class of suppressor mutants maintains high c-di-GMP levels, but loses the WS phenotype because the downstream EPS target (Pel) is inactivated. In three pel transposon mutants carrying insertions in pelA (PFLUOLIPICF7_RS06785), pelD (PFLUOLIPICF7_RS06770), and pelG (PFLUOLIPICF7_RS06755) - the majority of the transposon was removed by Cre-mediated recombination, eliminating possible polar effects. The Cre-deletion genotypes gave smooth colonies and were unable to form 24-h mats (Figure 3B-C), strongly indicating that at least three of the seven pel genes are essential for the PICF7-WS phenotype. We conclude that Pel is an important, c-di-GMP regulated, structural component of the PICF7-WS mat. 
A

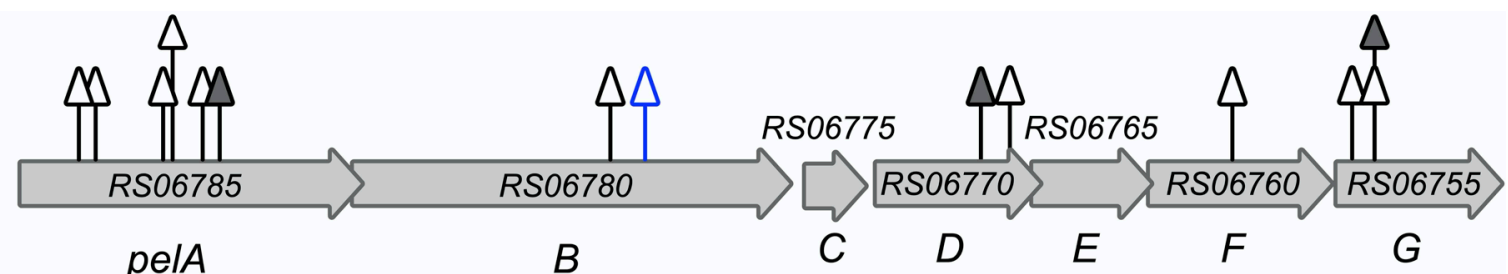
$\sim 1 \mathrm{~kb}$

pelA

PICF7

B

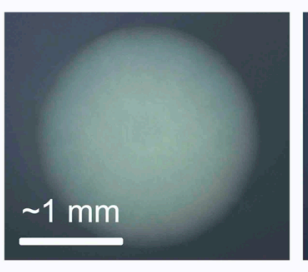

C

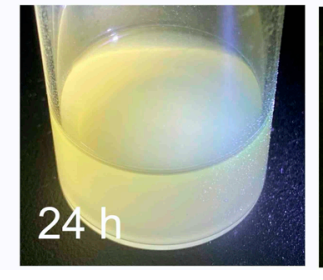

B

$F$

$G$
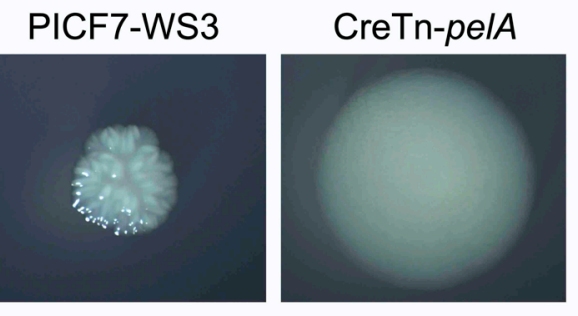

CreTn-pelD

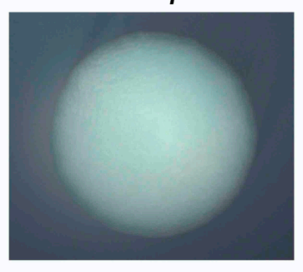

CreTn-pe/G
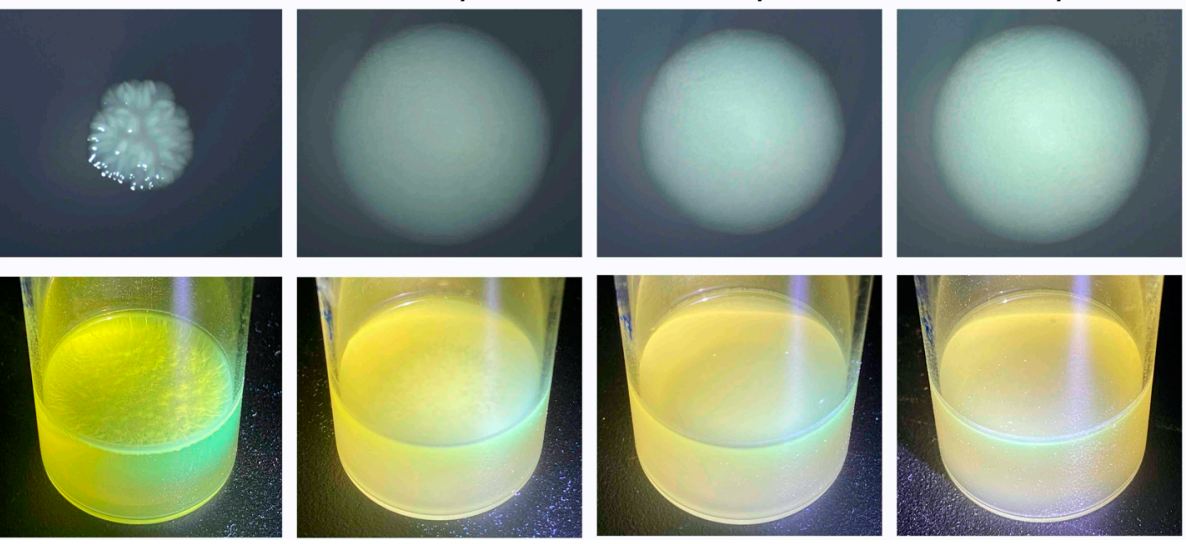

Figure 3. Pel production is a major structural component of the PICF7-WS phenotype. (A) Fourteen

independent WS suppressors were obtained in five of the seven genes of the PICF7 pel locus (pelA-G; Tables

$\mathrm{S} 2$ and S4). Grey triangles=transposon mutants obtained in the background of a $w s p F$ mutation (PICF7-WS3,

PICF7-WS8); blue triangle=transposon mutant obtained in the background of the aws $X$ mutation (PICF7-WS4);

open triangles=mutants carrying the full transposon; solid triangles $=$ mutants in which a Cre-mediated deletion of the original transposon has been constructed (giving CreTn-pelA, CreTn-pelD, CreTn-pelG). The Cre-deleted transposon mutants have lost the WS colony morphology on KB agar (B), and no longer form strong mats in static microcosms within 24 hours $(\mathbf{C})$. Brightness and exposure of some images were altered in Preview.

The A506-Web colony phenotype is semi-heritable

In contrast to SBW25-WS and PICF7-WS, the A506-Web phenotype is only semi-heritable. Firstly, A506-Web colonies derived from 72-hour static microcosms rapidly and repeatedly give rise to a mixture of web and smooth (i.e., wild type-like) colonies when plated on KB agar (Figure 4A). Secondly, when A506-Web colonies were grown in shaken overnight culture (i.e., an environment that precludes mat formation) and plated on $\mathrm{KB}$ agar, between $9 \%$ and $45 \%$ of colonies reverted to the ancestral, smooth morphotype (Figure 4B). No such colony diversity was observed arising from the colonies of the stable mat-forming morphotypes (A506-wspF-LoF, SBW25-WS, PICF7-WS, PICF7-LSm, or PICF7-SD) (Figure 4B). Of the web and smooth colony types arising from A506Web, only the web type retained the ability to give rise to 24 -h mats in static microcosms (Figure 4C).

The relative ease with which the A506-Web phenotype reverts to the wild-type morphotype suggests that, unlike PICF7-WS and SBW25-WS, it is not underpinned by a stable genetic change. Indeed, no SNPs or indels were confirmed on the A506 chromosome or plasmid during whole 
genome re-sequencing of the ten independent A506-Web isolates (Table S3). Interestingly, evidence of large-scale, tandem duplication events was found in all ten A506-Web isolates, suggesting a flexible region of the chromosome to one side of the replication terminus (Text S2). The affected regions vary in size $(\sim 158 \mathrm{~kb}$ to $\sim 634 \mathrm{~kb}$ ) and precise location (between genomic positions $1,900,000$ bp and 2,897,000 bp), but contain a shared segment of $\sim 126 \mathrm{~kb}$ beginning at genomic position $\sim 2,314,000 \mathrm{bp}$. While intriguing, these amplifications are not thought to contribute to the web phenotype, because (i) a similar region was observed upon sequencing the A506 wild-type, which had not been subjected to growth in a static microcosm, and (ii) the set of $\sim 115$ genes encoded in the shared region does not include any genes with known roles in polymer synthesis or regulation (e.g., pga, psl, wsp, aws, mwsR).

A
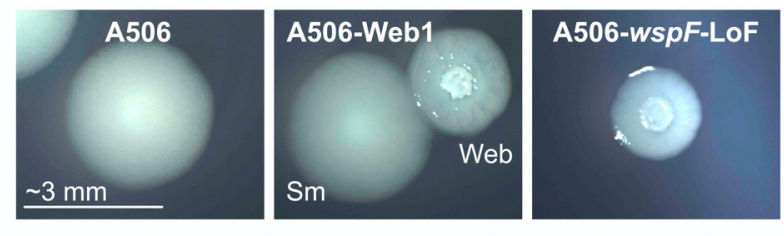

$\mathbf{B}$

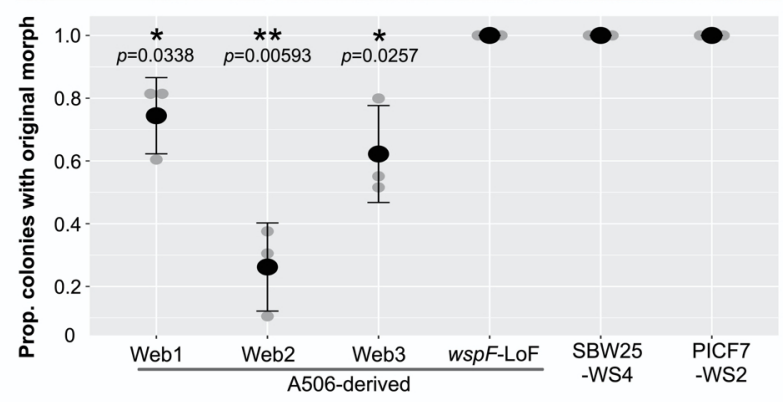

C
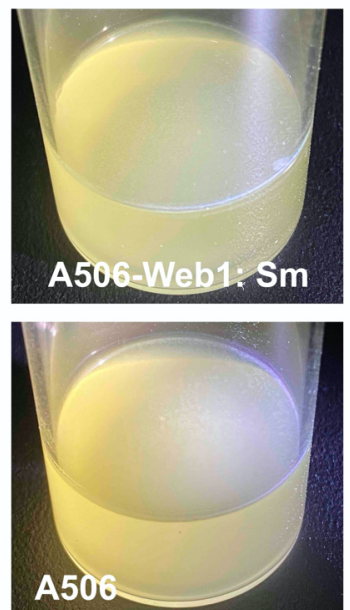
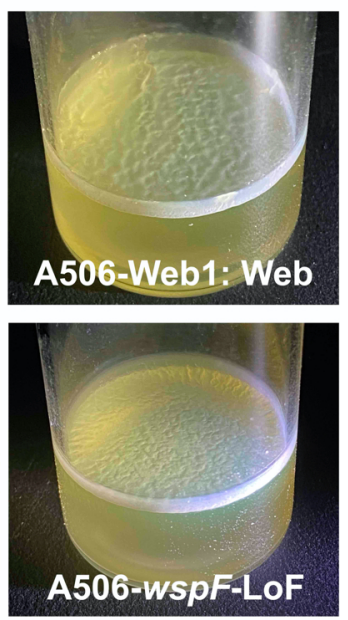

Isolate or strain

Figure 4. Semi-heritable (A506-Web) and heritable (A506-wspF-LoF) routes to mat formation in A506.

(A) A506-Web isolates from 72-h static microcosms give rise to a mixture of smooth ( $\mathrm{Sm}$ ) and web colonies on $\mathrm{KB}$ agar (A506-Web1 is pictured as an example). A506-wspF-LoF, carrying a loss-of-function mutation in $w s p F$, consistently generates colonies with a similar, web-like appearance. (B) Quantification of colony morphotype loss demonstrated significant degree of web morphotype loss in A506-Web colonies (one-tailed, one sample $t$-test $p<0.0338$ ), no loss of the web-like morphotype in A506-wspF-LoF, and no loss of WS morphotype in SBW25-WS4 or PICF7-WS2 (both carrying loss-of-function wspF mutations). Three biological replicates were performed for each strain, with a minimum of 45 colonies counted across three plates per replicate. Grey dots are individual data points, black dots are means, and black whiskers are standard errors. (C) Like A506, smooth colonies isolated from A506-Web1 in panel A (A506-Web1:Sm) do not give rise to a mat in 24-h static microcosms. Both the web colony counterparts (A506-Web1:Web) and A506-wspF-LoF form a strong mat within 24 hours. Brightness and exposure of some images were altered in Preview. 
these loci could generate differences in colony morphology, and 24-h mats. To investigate, a scarfree, loss-of-function mutation was constructed in the A506 wspF gene ( $\Delta 151-165$ in PflA506_1189, leading to the deletion of amino acids 51-55; see Text S3). This mutation is a commonly occurring loss-of-function mutation underpinning the SBW25-WS phenotype (Bantinaki et al., 2007; Gallie et al., 2019), and was also identified in SBW25-WS4 of this study. The resulting genotype, A506-wspFLoF, forms colonies that are similar in appearance to A506-Web colonies ("web-like"), and gives rise to 24-h mats (Figure 4A, 4C). Further, the web-like colony phenotype is stable; A506-wspF-LoF weblike colonies give rise to exclusively web-like colonies when streaked on agar plates, and when plated after growth in shaken overnight culture (Figure 4B). Hence, A506 carries the genetic and molecular machinery required to produce mats by at least one of the same mutational routes as SBW25-WS and PICF7-WS (loss-of-function mutations in $w s p F$ ). However, the emergence of mat formation by a readily reversible route appears to be favoured in our experiments.

\section{Pga and Psl contribute to A506 mat formation}

Molecular routes to mat formation by A506 are expected to culminate in the over-production of EPS (and/or other surface components). There are at least three candidate EPSs: Pga, Psl, and alginate (Table 1). Two of these, Pga and Psl, have been shown to be regulated by c-di-GMP in other pseudomonads (Borlee et al., 2010; Malone et al., 2010; Lind et al., 2017). We initially focused on Pga, because A506-Web colonies share morphological similarities with the SBW25 Pga wrinkly spreader (PWS). PWSs are WS-like genotypes, often carrying aws or $m w s R$ mutations, isolated from microcosms inoculated with an SBW25 strain deficient in cellulose biosynthesis (SBW25- $\Delta$ wss) (Lind et al., 2017).

To test the possibility that Pga plays a role in heritable mat formation, the four-gene $p g a$ operon (pgaA-D; PflA506_0154 to PflA506_0157) was cleanly deleted from A506-wspF-LoF, and the effect on colony and mat forming phenotypes assessed (Figure 5A). Elimination of Pga noticeably altered heritable mat formation; A506-wspF-LoF- $\Delta p g a$ formed visibly weaker 24-h mats than A506$w s p F-L o F$, and 72-h mats with a different texture. Deletion of pga from A506 had no immediately obvious phenotypic effects (Figure 5B), suggesting that while Pga may play a structural role in A506 mat formation, other EPSs can fulfill this role if required (perhaps in a similar manner to Pga substituting for cellulose in mats derived from SBW25- $\Delta$ wss).

The role of various structural components in mat formation by the emergent A506-Web type is not easy to elucidate, due to the semi-heritable nature of the phenotype; the phenotype is quickly lost during the successive rounds of growth required for the transposon mutagenesis and/or the genetic engineering protocol. Therefore, we deleted the pga (pgaA-D; PflA506_0154 to PflA506_0157) and psl (pslA-K; PflA506_1982 to PflA506_1971) loci, separately and in combination, from wild-type A506 (giving A506- $\Delta p g a$, A506- $\Delta p s l$, and A506- $\Delta p g a \Delta p s l$ ). 
1 Elimination of both $p g a$ and $p s l$ altered, but did not entirely eliminate, the emergence of 72-h mats

2 from A506 (Figure 5B). Overall, the results in this section indicate that while Pga and Psl play a role

3 in mat formation, other structural components may also contribute to the emergence of A506 mats. 4

A
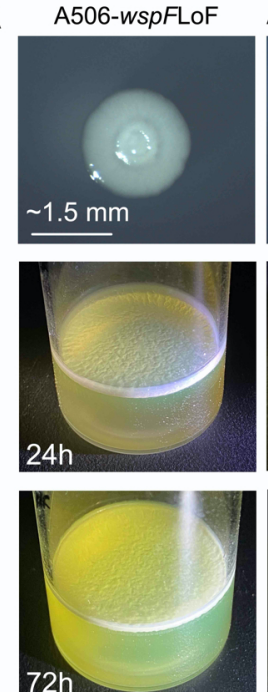

A506-wspFLoF- $\triangle p g$ a
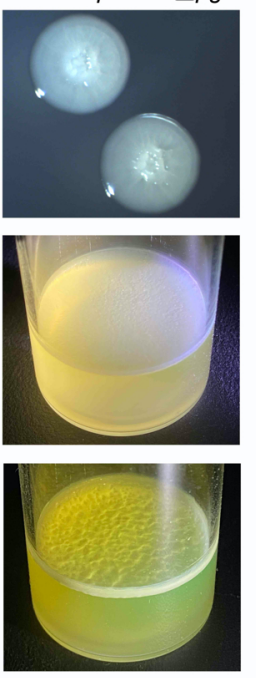

A506
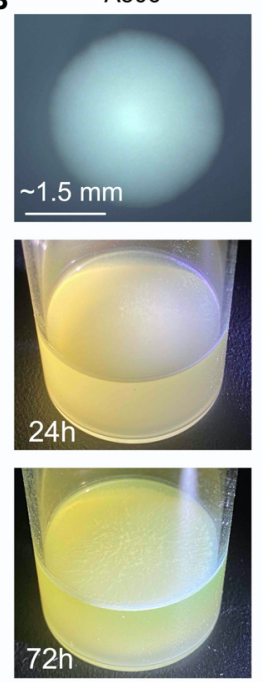

A506- $\triangle p g a$
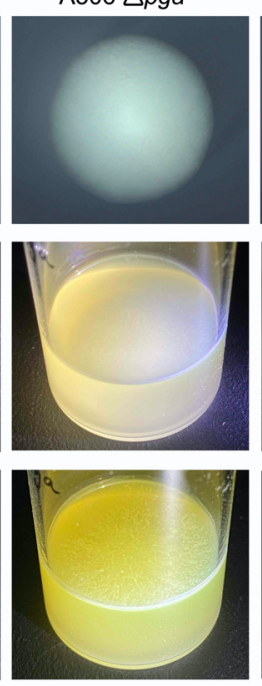

A506- $\triangle p s l$
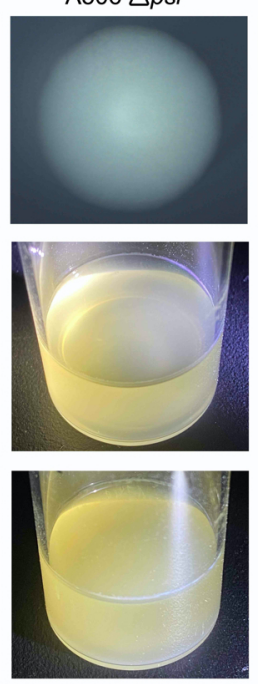

A506- $\triangle \mathrm{pga} \triangle \mathrm{psl}$
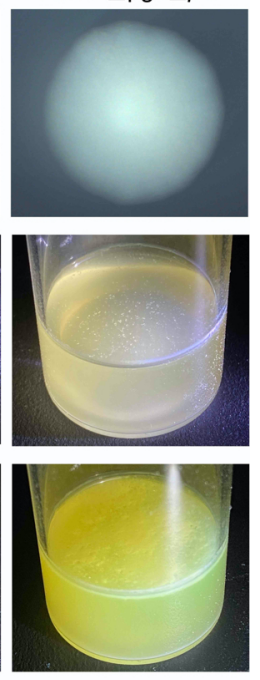

6

Figure 5. Pga and Psl contribute to A506 mat formation. (A) The effect of scar-free deletion of the pga operon from A506-wspF-LoF on colony morphology (top), 24-h mat formation (middle), and 72-hour mat formation (bottom). (B) Same as panel A, but with pga, psl, or both deleted from A506. All photographs within a single row were taken under the same conditions, at the same time point. Brightness and exposure of some images were altered in Preview.

\section{DISCUSSION}

P. fluorescens SBW25 readily acquires mutations in wsp, aws, or $m w s R$ that elevate c-di-GMP, leading to the constitutive synthesis of cellulose and the emergence of mats at the air-liquid interface. Here, we have investigated the divergent mechanisms by which SBW25 relatives $P$. simiae PICF7 and $P$. fluorescens A506 produce similar, emergent mats. The details of mat formation in PICF7 and A506 are discussed below, and a summary model is provided in Figure 6.

\section{A model for mat formation by PICF7-WS}

One morphotype that contributes to the emergence of mats from PICF7 is the wrinkly spreader (PICF7-WS). There are notable parallels between PICF7-WS and SBW25-WS. Namely, (i) they produce similar-looking, heritably stable, wrinkly colonies and 24-h mats, and (ii) these phenotypes are underpinned by c-di-GMP-elevating mutations in $w s p$, aws, or $m w s R$ homologues. However, the downstream targets of c-di-GMP differ in the two strains. In SBW25, elevated c-di-GMP levels stimulate the mass production of cellulose by the Wss biosynthetic machinery (Spiers et al., 2002; Gal et al., 2003; Goymer et al., 2006). PICF7 lacks wss homologues, and cannot synthesize cellulose. Instead, the PICF7-WS phenotype requires the over-expression of Pel (Figure 6). 
Pel is a cationic, glucose-rich EPS that has mainly been characterized as a structural component of $P$. aeruginosa biofilms (Jennings et al., 2015). In P. aeruginosa, c-di-GMP-mediated over-production of Pel is required for the emergence of WS-like, rugose small-colony variants (RSCVs) (D'Argenio et al., 2002; Hickman et al., 2005; Starkey et al., 2009). C-di-GMP-mediated regulation of $P$. aeruginosa Pel production occurs through FleQ, a c-di-GMP binding transcriptional regulator. FleQ binds to two stretches of DNA upstream of pelA, and, in the absence of c-di-GMP, represses pel transcription (Hickman and Harwood, 2008). When DNA-bound FleQ interacts with cdi-GMP, the conformation of the region changes such that FleQ becomes a transcriptional activator of pel (Baraquet et al., 2012; Matsuyama et al., 2016). FleQ is also involved in c-di-GMP dependent EPS production in Pseudomonas putida (Ramos-González et al., 2016; Molina-Henares et al., 2017).

The above suggests that FleQ may play a role in c-di-GMP mediated regulation of Pel production in PICF7. Indeed, a recent study reported that a PICF7 strain carrying an inactivating insertion in fleQ (PFLUOLIPICF7_16515) resulted in altered capacity to form a biofilm (MontesOsuna et al., 2021). The extent to which, and mechanisms whereby, fle $Q$ and other regulators are involved in PICF7 Pel regulation remain to be elucidated.

\section{Additional routes to mat formation in PICF7}

In addition to WS, 72-h PICF7 microcosms were typically observed to contain multiple other emergent colony morphotypes (LSm, SD, LD). Each morphotype has its own distinguishing phenotypic features, which presumably result from different molecular processes. With the exception of WS, these molecular processes remain almost entirely uncharacterized; they may or may not involve c-di-GMP, and may each regulate the expression of different downstream EPSs or cell surface components. We do know that at least three of the emergent morphotype classes - WS, LSm and SD - are heritable (i.e., are likely underpinned by stable mutations). In addition, these three morphotypes can each independently form 24-h mats (see Figure S1), suggesting that each type arises and competes for dominance in the emerging, 72-h mat. The relative proportions of each morphotype have not yet been studied, but these are likely to depend on a combination of the rate at which each morphotype arises (e.g., mutational target size and localized mutation rates), and the relative fitness of each type in the emerging population.

The co-emergence of multiple, distinct mat-forming morphotypes strongly suggests that 72-h PICF7 mats consist of phenotypically and genotypically heterogeneous subpopulations, possibly with each morphotype producing distinct EPSs, and/or cell surface components. PICF7 has the genetic capacity to produce several EPSs (including Pel, Pga, Psl, and alginate), and various cell surface components (e.g., adhesins, lipopolysaccharides, pili). Expression of each of these components is expected to confer specific physicochemical properties on the surrounding cells (reviewed in Rehm, 2010), some of which may be beneficial in the natural plant environment. 
Molecular routes to semi-heritable mat formation by A506-Web

2 The emergence of A506 mats over 72 hours is underpinned by the semi-heritable A506-Web

3 morphotype, which forms colonies with a web-like internal structure that can form full mats within 24

4 hours. The A506-Web phenotype is less stable than the mat-forming morphotypes arising from PICF7 and SBW25. The degree of morphotype stability provides some insight into the underlying molecular causes of the phenotype. At one end of the scale, a readily reversible phenotype is likely to be underpinned by non-heritable mechanisms (e.g., activation of an EPS transcription factor in the presence of a particular environmental stimulus; see introduction). Such mechanisms can be switched on and off within the lifetime of a cell, and are thus not heritable. At the opposite end of the scale, an (almost) irreversible phenotype is likely to be underpinned by a stable genetic change (e.g., a deletion in $w s p F$ giving rise to the WS phenotype). In the absence of further mutation(s), the resulting phenotype is stable and heritable. The semi-heritable A506-Web phenotype is not expected to result from either a straightforward transcriptional change (no heritability), or a stable genetic change (heritability), but a mechanism that generates semi-heritability.

We see two hypothetical possibilities for a mechanism underpinning semi-heritability (Figure 6). Firstly, the A506-Web phenotype could conceivably result from a non-stable genetic change that flips between two states at relatively high frequency (i.e., phase variation). These types of mutations examples of which include slippage in homopolymeric tracts (De Bolle et al., 2000; Orsi et al., 2010), site-specific inversions (Abraham et al., 1985; Dybvig and Yu, 1994), and tandem duplication events (Anderson and Roth, 1981; Ayan et al., 2020) - occur and revert randomly, but at frequencies several orders of magnitude higher than standard SNPs (reviewed in Anderson and Roth, 1977; Moxon et al., 2006). If such a high-frequency mutational locus were to exist within, for example, an A506 EPS biosynthetic locus, an initially clonal, growing population would rapidly and repeatedly generate subpopulations of "EPS on" (web) and "EPS off" (wild-type). Once both forms exist, selection would act to influence the relative proportion of each form, with web and wild-type dominating in static and shaken environments, respectively. While we did not find evidence of mutable loci underpinning the unstable A506-Web phenotype, we note that many kinds of high-frequency mutations are notoriously difficult to identify using whole genome re-sequencing. However, the rate at which the A506-Web morphotype repeatedly reverted to the wild-type morphotype in overnight shaken culture was observed to be much higher than would typically be expected for phase variants (Anderson and Roth, 1977; Moxon et al., 2006).

A second possibility is that no genetic change is required to switch between A506-Web and wild-type morphotypes. Instead, semi-heritability may be achieved by a molecular network topology that can generate two distinct, semi-stable phenotypic states ("EPS on" or "EPS off") (Tiwari et al., 2011). To illustrate, imagine the following example. In the static microcosm, a signal activates expression of a transcription factor (TF), which in turn activates the transcription of (i) a DGC and/or EPS biosynthetic genes, and (ii) itself. This leads to (i) the "EPS on" state and mat formation, and (ii) 
self-perpetuating TF production that can persist even after the initial environmental stimulus is removed. A switch back to the "EPS off" state requires TF levels to stochastically drop below a particular threshold, perhaps as a result of stochastic division of intracellular protein content upon cell division. Examples of bistable molecular networks have been shown to underpin semi-heritable, ON/OFF expression of colanic acid-like capsules in P. fluorescens SBW25 (Gallie et al., 2015; Gallie et al., 2019; Remigi et al., 2019), bistable expression of the PDE PdeL in E. coli (Reinders et al., 2016), and bistable white-opaque switching in Candida albicans (Zordan et al., 2006). Some bistable switches can switch between states more rapidly than is achieved by phase variation (e.g., Gallie et al., 2015; Remigi et al., 2019), and hence could conceivably be involved in the A506-Web phenotype. The existence of a semi-heritable mechanism underpinning A506 mat formation provides a degree of phenotypic flexibility for A506 that has - at least so far - not been observed in laboratory populations of SBW25 and PICF7. Such a mechanism may enable A506 to readily and repeatedly switch between planktonic and sessile lifestyles, without the accumulation of irreversible, loss-offunction mutations in EPS biosynthetic pathways.

\section{A stable route to A506 mat formation: wspF mutations}

In addition to the semi-heritable route, A506 can form stable web-like colonies and mats through at least one of the three mutational routes that underpin the SBW25-WS and PICF7-WS phenotypes: loss-of-function mutations in $w s p F$, a negative regulator of the WspR DGC (Figure 6). Protein domain predictions for the Wsp pathway (see Table S2) are consistent with $w s p F$ mutations having similar downstream effects in all three strains: de-repression of WspR DGC activity, leading to an increase in c-di-GMP, and overproduction of at least one EPS (cellulose in SBW25, Pel in PICF7, and Pga in A506). Wsp-mediated control of these three distinct EPSs demonstrates a certain degree of evolutionary flexibility in mat structural components, indicating that EPS biosynthetic loci can be lost or gained, and may be readily incorporated into existing host regulatory networks.

It is currently unclear to what extent the web-like phenotype generated by mutation of $w s p F$ resembles the semi-heritable A506-Web phenotype (at both molecular and structural levels).

However, while many mutational routes to mat formation are presumably available in A506 (i.e., loss of function mutations in $w s p F$ ), our results indicate that genotypes carrying these mutations do not dominate in emergent mats; all ten of our independently-isolated A506-Web types showed the semiheritable web-like phenotype, and no $w s p F$ (or other) mutations were identified. The bias towards the semi-heritable route to mat formation may result from a higher rate of A506-Web generation than $w s p F$ mutation, and/or a selective advantage of A506-Web over wspF mutants. 


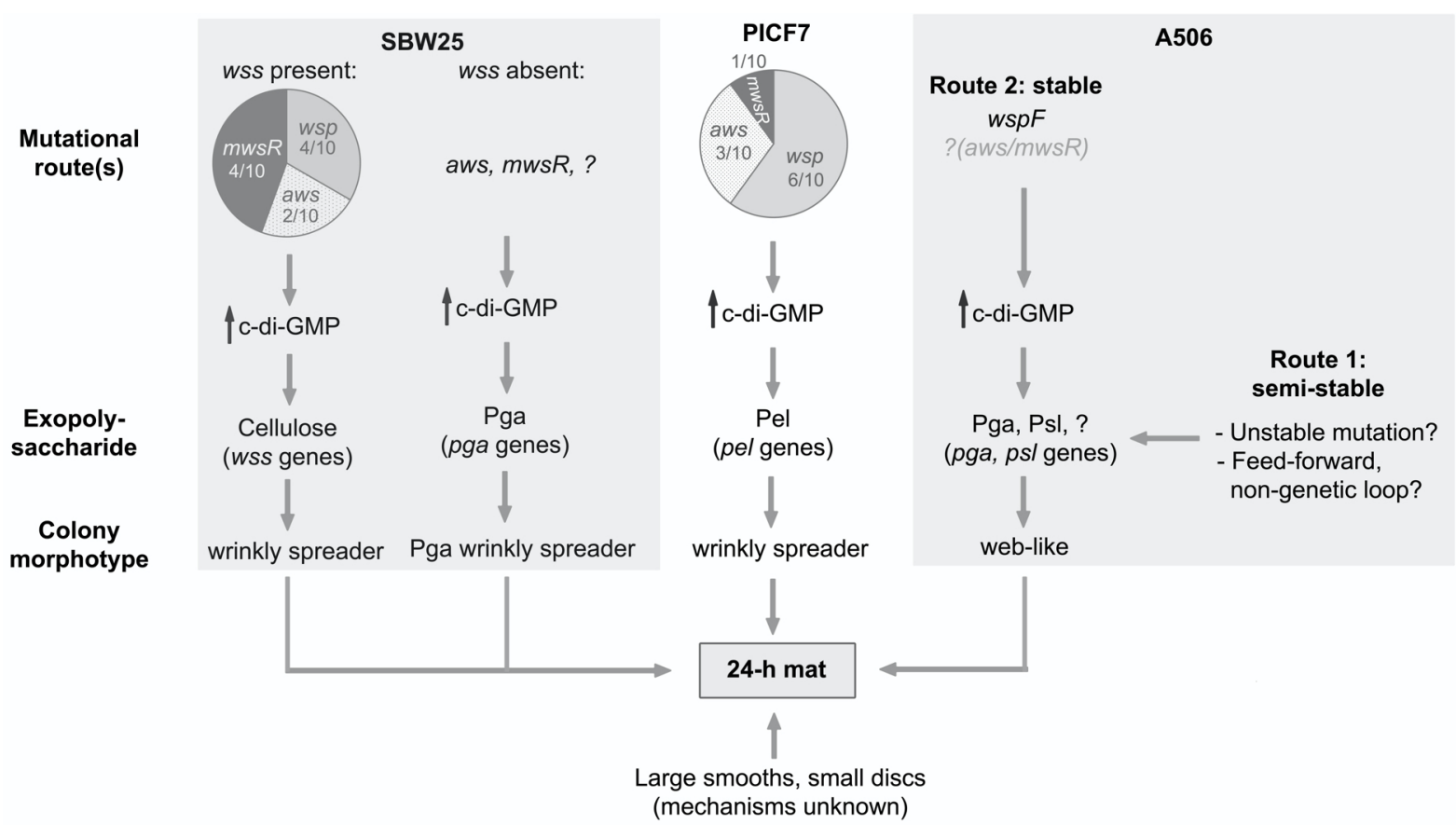

Figure 6. Model of the molecular routes to 72-h mat formation in P. simiae PICF7 and P. fluorescens A506, as compared with the previously well-studied $P$. fluorescens SBW25. Pie charts were drawn using mutations identified by genome sequencing of ten SBW25 and ten PICF7 WS genotypes (see Table 2).

The diversity of molecular and structural routes to mat formation in P. fluorescens SBW25, P. simiae PICF7, and P. fluorescens A506 demonstrates a high degree of evolutionary flexibility in EPS regulation, highlighting the ecological and evolutionary importance of mat and biofilm formation.

\section{EXPERIMENTAL PROCEDURES}

Phylogenetic tree construction

The complete genome sequences of ten close Pseudomonas strains were downloaded from NCBI in February 2021 (for reference numbers see Table S1). The most divergent strain, Pseudomonas 14 fluorescens UK4, was used as an outgroup. A sequence alignment was generated using REALPHY version 1.13 (Bertels et al., 2014). In REALPHY, three reference alignments were generated (A506, 6 SBW25, and RE*-1-1-14) and merged. The merged alignment consisted of 1,464,229 alignment 17 columns. From this alignment, a maximum likelihood tree was generated with PHYML (GTR substitution model) (Guindon and Gascuel, 2003), and visualized in Geneious (v11.1.4).

\section{Strains, plasmids, and primers}

Details of all strains, plasmids, and oligonucleotides used in this study are provided in Table S1. 
1

2

3

\section{Growth conditions}

E. coli cloning strains were grown in Lysogeny Broth (LB) at $37^{\circ} \mathrm{C}$ ( $\sim 18$ hours, shaking). When not grown in static microcosms (see below), Pseudomonas strains were grown in King's Medium B (KB; King et al., 1954) at $28^{\circ} \mathrm{C}$ ( $\sim 18$ hours, shaking). Pseudomonas cells were plated on KB containing 1.5 $\%$ agar, and incubated at $28^{\circ} \mathrm{C}$ for 48 hours. Where appropriate, media were supplemented with: nitrofurantoin (NF; $100 \mu \mathrm{g} \mathrm{ml}^{-1}$ ), kanamycin $\left(\mathrm{Km} ; 50 \mu \mathrm{g} \mathrm{ml}^{-1}\right.$ ), tetracycline $\left(\mathrm{Tc} ; 50 \mu \mathrm{g} \mathrm{m}{ }^{-1}\right.$ ), and/or 5Bromo-4-Chloro-3-Indolyl ß-D-Galactopyranoside (X-gal; $60 \mu \mathrm{g} \mathrm{ml}^{-1}$ ).

\section{Mat formation in static microcosms}

Static microcosms were grown in $30 \mathrm{ml}$ glass tubes containing $6 \mathrm{ml} \mathrm{KB}$. Each microcosm was inoculated with $6 \mu$ of stationary phase Pseudomonas culture, vortexed for 5 seconds, the lid loosened, and grown without agitation at $28^{\circ} \mathrm{C}$ for 24 or 72 hours. For analysis of emergent colony morphotypes, microcosms were vortexed for 1 minute and dilution plated on KB agar.

\section{Photography}

Colonies were visualized under a Leica MS5 dissection microscope, and photographed with a VisiCam ${ }^{\circledR} 1.3$ (VWR International). Microcosm photographs were taken with an iPhone 11. Photographs were cropped and, where noted in figure legends, the exposure and/or brightness uniformly altered in Preview (v11.0).

\section{Whole genome sequencing}

Single colony isolates were grown to stationary phase in liquid KB (shaking) and genomic DNA isolated with a Qiagen DNeasy Blood and Tissue Kit (Qiagen). Extracted genomic DNA was checked for quality using agarose gel electrophoresis and a Nanodrop ${ }^{\mathrm{TM}} 3300$ Fluorospectrometer. Whole genome re-sequencing was subsequently performed by the sequencing facility at the Max Planck Institute for Evolutionary Biology (Ploen, Germany), in three separate runs. The first run aimed to identify mutations in thirty SBW25-WS, PICF7-WS, and A506-Web isolates. $300 \mathrm{bp}$, paired-end reads were generated with a MiSeq Reagent Kit v3 (Illumina). Usable data was generated for 29 of 30 isolates; PICF7-WS5 was unsuccessful. PICF7-WS5 was re-sequenced in a second run, during which $150 \mathrm{bp}$, paired end reads were generated with a NextSeq 550 Output v2.5 Kit (Illumina). A third run was used to generate high coverage reads for A506 wild-type and one A506-Web isolate (A506Web5). 150 bp, paired-end reads were generated with a NextSeq 550 Output v2.5 kit (Illumina). All sequencing reads are available at NCBI sequence read archive (Leinonen et al., 2011), under BioProject number XXXXX-YYYYYY. 
Analysis of whole genome sequencing data for PICF7-WS and SBW25-WS

2 A minimum of 1 million reads per isolate were aligned to the PICF7 (GenBank CP005975.1;

3 Martínez-García et al., 2015) or SBW25 (RefSeq NC_012660.1; Silby et al., 2009) reference genome

4 sequences, using breseq (Deatherage and Barrick, 2014). A minimum mean coverage of 26.3 and 28.9

5 was obtained for the PICF7-WS and SBW25-WS genotypes, respectively. A list of possible mutations was generated for each genome (Table S3), and unique mutations were confirmed in the genome of interest by PCR amplification and Sanger sequencing. In isolates where no unique candidate mutations were initially identified by breseq (SBW25-WS6 and SBW25-WS8), WS mutations were identified by PCR amplification and Sanger sequencing of commonly mutated loci in WS types. For a detailed list of the WS-causing mutations, see Text S1.

Analysis of whole genome sequencing data for A506

The first attempt at re-sequencing A506-Web isolates resulted in the prediction of $>200$ mutations per isolate by breseq, many of which were present in all ten isolates (Table S3). To clarify which, if any, of these predicted mutations were real and relevant for the Web phenotype, we sequenced our copy of A506 wild type and one Web isolate (A506-Web5) at high coverage. For each of these two samples, slightly under 6 million $150 \mathrm{bp}$, paired-end sequencing reads were obtained, and aligned to the NCBI reference sequences for the A506 chromosome (NC_017911.1; Loper et al., 2012) and plasmid (NC_021361.1; Stockwell et al., 2013) using breseq (Deatherage and Barrick, 2014). Mean coverages of 144.9 and 139.9 (A506 wild type chromosome and plasmid, respectively), and 151.1 and 111.9 (A506-Web5 chromosome and plasmid, respectively) were obtained, and $\sim 100$ mutations were predicted in each sample. All differences predicted in A506-Web5 were also predicted in A506 wild type, and no convincing additional differences were found in the lower coverage genome resequencing in any of the other Web isolates (Table S3).

Transposon mutagenesis

Four $P$. fluorescens PICF7 WS genotypes (WS3, WS4, WS7, WS8; carrying mutations in wspF, aws $X, m w s R, w s p F$, respectively) were subjected to a suppressor analysis via random mutagenesis with the IS- $\Omega-\mathrm{Km}$-/hah transposon (Jacobs et al., 2003). The protocol previously described for the mutagenesis of $P$. fluorescens SBW25 (Giddens et al., 2007) was used, with the exception that PICF7-WS recipient genotypes were not subjected to a heat shock prior to biparental conjugation. loss of both the WS colony and 24-h mat forming phenotype. The transposon insertion site was determined in 100 suppressor mutants (Table S4). In selected transformants, the bulk of the transposon was deleted ("CreTn" types), leaving $189 \mathrm{bp}$ at the insertion site and eliminating polar effects (Giddens et al., 2007). 


\section{Colony morphotype stability test}

Single colonies of six strains (A506-Web1, A506-Web2, A506-Web3, A506-wspF-LoF, SBW25WS4, and PICF7-WS2) were grown from glycerol stocks on KB agar. For each strain, three single colonies displaying the Web or WS phenotype were grown overnight in liquid KB. Overnight cultures were vortexed, dilution plated on KB agar. The number and morphotype of colonies from each culture was recorded. For each culture, between 45 and 300 colonies were observed, across three KB plates. The proportion of colonies showing the original morphotype (Web or WS) was recorded, and the mean and standard error calculated (Figure 4B; Raw Data File 1). In the case of strains carrying a $w s p F$ mutation (A506-wspF-LoF, SBW25-WS4, PICF7-WS2), no variation in colony morphology was observed. Parametric, one-tailed, one-sample $t$-tests were used to detect loss of Web colony morphology in A506-Web types. Analyses were performed in R (v3.6.0). Significance levels $* 0.05<p<0.01, * * 0.01<p<0.001$.

\section{Genetic engineering in A506}

Scar-free deletions were constructed in A506 using a similar protocol to that outlined for SBW25 (Zhang and Rainey, 2007). Briefly, deletion fragments were constructed by SOE-PCR (Ho et al., 1989), ligated into the suicide vector pUIC3 (Rainey, 1999), and delivered into the relevant A506 background using a recombination-based, two-step allelic exchange protocol. The differences to the SBW25 protocol are (i) A506 was not subjected to a heat shock prior to the first recombination event, (ii) a higher concentration of Tc was used $\left(50 \mu \mathrm{g} \mathrm{ml}^{-1}\right)$ to select first recombinants, and (iii) cycloserine enrichment was not used to enrich for second recombinants; first recombinant types were simply grown in flasks and plated onto LB+Xgal agar to identify colonies that had lost the pUIC3 vector (i.e. white, Tc sensitive colonies). Presence of the deletion was confirmed using PCR and Sanger sequencing with primers outside the manipulation region. In each case, the final result is a strain in which the target sequence is cleanly deleted, leaving no trace of the pUIC3 vector. See Text S3 for more detail on each genotype, and the engineering process.

\section{ACKNOWLEDGEMENTS}

We thank Paul Rainey, Jesús Mercado-Blanco, and Steven Lindow for the kind gifts of $P$. fluorescens SBW25, P. simiae PICF7, and P. fluorescens A506, respectively. We thank Frederic Bertels for assistance with building the Pseudomonas phylogenetic tree, and Gunda Dechow-Seligmann for technical assistance. Funds were received from the Max Planck Society, and the International Max Planck Research School for Evolutionary Biology (IMPRS-EB). 
1

2

\section{REFERENCES} manuscript. 1088. $1500-1516$.

\section{AUTHOR CONTRIBUTIONS}

$\mathrm{AM}$ and JG conceived the research, performed the experiments and data analyses, and wrote the

Abraham, J.M., Freitag, C.S., Clements, J.R., and Eisenstein, B.I. (1985). An invertible element of DNA controls phase variation of type 1 fimbriae of Escherichia coli. Proceedings of the National Academy of Sciences of the United States of America, 82: 5724-5727.

Amikam, D., and Galperin, M.Y. (2006). PilZ domain is part of the bacterial c-di-GMP binding protein. Bioinformatics, 22: 3-6.

Anderson, P., and Roth, J. (1981). Spontaneous tandem genetic duplications in Salmonella typhimurium arise by unequal recombination between rRNA (rrn) cistrons. Proceedings of the National Academy of Sciences of the United States of America, 78: 3113-3117.

Anderson, R.P., and Roth, J.R. (1977). Tandem genetic duplications in phage and bacteria. Annual Review of Microbiology, 31: 473-505.

Ayan, G.B., Park, H.J., and Gallie, J. (2020). The birth of a bacterial tRNA gene by large-scale, tandem duplication events. eLife, 9: e57947.

Bantinaki, E., Kassen, R., Knight, C.G., Robinson, Z., Spiers, A.J., and Rainey, P.B. (2007). Adaptive divergence in experimental populations of Pseudomonas fluorescens. III. Mutational origins of wrinkly spreader diversity. Genetics, 176: 441-453.

Baraquet, C., Murakami, K., Parsek, M.R., and Harwood, C.S. (2012). The FleQ protein from Pseudomonas aeruginosa functions as both a repressor and an activator to control gene expression from the pel operon promoter in response to c-di-GMP. Nucleic Acids Research, 40: 7207-7218.

Barrick, J.E., Colburn, G., Deatherage, D.E., Traverse, C.C., Strand, M.D., Borges, J.J., et al. (2014). Identifying structural variation in haploid microbial genomes from short-read resequencing data using breseq. BMC Genomics, 15: 1039.

Bertels, F., Silander, O.K., Pachkov, M., Rainey, P.B., and Nimwegen, E. Van (2014). Automated reconstruction of whole-genome phylogenies from short-sequence reads. Molecular Biology and Evolution, 31: 1077-

Boehm, A., Steiner, S., Zaehringer, F., Casanova, A., Hamburger, F., Ritz, D., et al. (2009). Second messenger signalling governs Escherichia coli biofilm induction upon ribosomal stress. Molecular Microbiology, 72:

Borlee, B.R., Goldman, A.D., Murakami, K., Samudrala, R., Wozniak, D.J., and Parsek, M.R. (2010). Pseudomonas aeruginosa uses a cyclic-di-GMP-regulated adhesin to reinforce the biofilm extracellular matrix. Molecular Microbiology, 75: 827-842.

Chan, C., Paul, R., Samoray, D., Amiot, N.C., Giese, B., Jenal, U., and Schirmer, T. (2004) Structural basis of activity and allosteric control of diguanylate cyclase. Proceedings of the National Academy of Sciences of the United States of America, 101: 17084-17089.

Chen, A.G.Y., Sudarsan, N., and Breaker, R.R. (2011). Mechanism for gene control by a natural allosteric group I ribozyme. $R N A$, 17: 1967-1972. 
Colvin, K.M., Irie, Y., Tart, C.S., Urbano, R., Whitney, J.C., Ryder, C., et al. (2012). The Pel and Psl polysaccharides provide Pseudomonas aeruginosa structural redundancy within the biofilm matrix. Environmental Microbiology, 14: 1913-1928.

Costerton, J.W. (1999). Bacterial biofilms: a common cause of persistent infections. Science, 284: 1318-1322.

D’Argenio, D.A., Calfee, M.W., Rainey, P.B., and Pesci, E.C. (2002). Autolysis and autoaggregation in Pseudomonas aeruginosa colony morphology mutants. Journal of Bacteriology, 184: 6481-6489.

Davey, M.E., and O’toole, G.A. (2000). Microbial biofilms: from ecology to molecular genetics. Microbiology and Molecular Biology Reviews, 64: 847-867.

De Bolle, X., Bayliss, C.D., Field, D., Ven, T. van de, Saunders, N.J., Hood, D.W., and Moxon, E.R. (2000). The length of a tetranucleotide repeat tract in Haemophilus influenzae determines the phase variation rate of a gene with homology to type III DNA methyltransferases. Molecular Microbiology, 35: 211-222.

Deatherage, D.E., and Barrick, J.E. (2014). Identification of mutations in laboratory-evolved microbes from nextgeneration sequencing data using breseq. Methods in Molecular Biology, 1151: 165-188.

Duerig, A., Abel, S., Folcher, M., Nicollier, M., Schwede, T., Amiot, N., et al. (2009). Second messengermediated spatiotemporal control of protein degradation regulates bacterial cell cycle progression. Genes \& Development, 23: 93-104.

Dybvig, K., and Yu, H. (1994). Regulation of a restriction and modification system via DNA inversion in Mycoplasma pulmonis. Molecular Microbiology, 12: 547-560.

Friedman, L., and Kolter, R. (2004). Genes involved in matrix formation in Pseudomonas aeruginosa PA14 biofilms. Molecular Microbiology, 51: 675-690.

Gal, M., Preston, G.M., Massey, R.C., Spiers, A.J., and Rainey, P.B. (2003). Genes encoding a cellulosic polymer contribute toward the ecological success of Pseudomonas fluorescens SBW25 on plant surfaces. Molecular Ecology, 12: 3109-3121.

Gallie, J., Bertels, F., Remigi, P., Ferguson, G.C., Nestmann, S., and Rainey, P.B. (2019). Repeated phenotypic evolution by different genetic routes in Pseudomonas fluorescens SBW25. Molecular Biology and Evolution, 36: 1071-1085.

Gallie, J., Libby, E., Bertels, F., Remigi, P., Jendresen, C.B., Ferguson, G.C., et al. (2015) Bistability in a metabolic network underpins the de novo evolution of colony switching in Pseudomonas fluorescens. PLoS Biology, 13: e1002109.

Giddens, S.R., Jackson, R.W., Moon, C.D., Jacobs, M.A., Zhang, X.X., Gehrig, S.M., and Rainey, P.B. (2007) Mutational activation of niche-specific genes provides insight into regulatory networks and bacterial function in a complex environment. Proceedings of the National Academy of Sciences of the United States of America, 104: 18247-18252.

Goymer, P., Kahn, S.G., Malone, J.G., Gehrig, S.M., Spiers, A.J., and Rainey, P.B. (2006). Adaptive Divergence in experimental populations of Pseudomonas fluorescens. II. Role of the GGDEF regulator WspR in evolution and development of the wrinkly spreader phenotype. Genetics, 173: 515-526.

Guindon, S., and Gascuel, O. (2003). A simple, fast, and accurate algorithm to estimate large phylogenies by maximum likelihood. Systematic Biology, 52: 696-704.

Hickman, J.W., and Harwood, C.S. (2008). Identification of FleQ from Pseudomonas aeruginosa as a c-di-GMPresponsive transcription factor. Molecular Microbiology, 69: 376-389. 
Hickman, J.W., Tifrea, D.F., and Harwood, C.S. (2005). A chemosensory system that regulates biofilm formation through modulation of cyclic diguanylate levels. Proceedings of the National Academy of Sciences of the United States of America, 102: 14422-14427.

Ho, S.N., Hunt, H.D., Horton, R.M., Pullen, J.K., and Pease, L.R. (1989). Site-directed mutagenesis by overlap extension using the polymerase chain reaction. Gene, 77: 51-59.

Jacobs, M.A., Alwood, A., Thaipisuttikul, I., Spencer, D., Haugen, E., Ernst, S., et al. (2003). Comprehensive transposon mutant library of Pseudomonas aeruginosa. Proceedings of the National Academy of Sciences of the United States of America, 100: 14339-14344.

Jennings, L.K., Storek, K.M., Ledvina, H.E., Coulon, C., Marmont, L.S., Sadovskaya, I., et al. (2015). Pel is a cationic exopolysaccharide that cross-links extracellular DNA in the Pseudomonas aeruginosa biofilm matrix. Proceedings of the National Academy of Sciences of the United States of America, 112: 1135311358.

King, E.O., Ward, M.K., and Raney, D.E. (1954). Two simple media for the demonstration of pyocyanin and fluorescin. The Journal of Laboratory and Clinical Medicine, 44: 301-307.

Lee, V.T., Matewish, J.M., Kessler, J.L., Hyodo, M., Hayakawa, Y., and Lory, S. (2007). A cyclic-di-GMP receptor required for bacterial exopolysaccharide production. Molecular Microbiology, 65: 1474-1484.

Leinonen, R., Sugawara, H., and Shumway, M. (2011). The sequence read archive. Nucleic Acids Research, 39: D19-D21.

Li, Z., Chen, J.-H., Hao, Y., and Nair, S.K. (2012). Structures of the PelD cyclic diguanylate effector involved in pellicle formation in Pseudomonas aeruginosa PAO1. Journal of Biological Chemistry, 287: 30191-30204.

Liang, Z.-X. (2015). The expanding roles of c-di-GMP in the biosynthesis of exopolysaccharides and secondary metabolites. Natural Product Reports, 32: 663-683.

Lind, P.A., Farr, A.D., and Rainey, P.B. (2017). Evolutionary convergence in experimental Pseudomonas populations. The ISME Journal, 11: 589-600.

Lind, P.A., Libby, E., Herzog, J., and Rainey, P.B. (2019). Predicting mutational routes to new adaptive phenotypes. eLife, 8: e38822.

Loper, J.E., Hassan, K.A., Mavrodi, D.V., Davis, E.W., Lim, C.K., Shaffer, B.T., et al. (2012). Comparative genomics of plant-associated Pseudomonas spp.: Insights into diversity and inheritance of traits involved in multitrophic interactions. PLoS Genetics, 8: e1002784.

Malone, J.G., Jaeger, T., Spangler, C., Ritz, D., Spang, A., Arrieumerlou, C., et al. (2010). YfiBNR mediates cyclic di-GMP dependent small colony variant formation and persistence in Pseudomonas aeruginosa. PLoS Pathogens, 6: e1000804.

Malone, J.G., Williams, R., Christen, M., Jenal, U., Spiers, A.J., and Rainey, P.B. (2007). The structure-function relationship of WspR, a Pseudomonas fluorescens response regulator with a GGDEF output domain. Microbiology, 153: 980-994.

Martínez-García, P.M., Ruano-Rosa, D., Schilirò, E., Prieto, P., Ramos, C., Rodríguez-Palenzuela, P., and Mercado-Blanco, J. (2015). Complete genome sequence of Pseudomonas fluorescens strain PICF7, an indigenous root endophyte from olive (Olea europaea L.) and effective biocontrol agent against Verticillium dahliae. Standards in Genomic Sciences, 10: 10. 
Matsuyama, B.Y., Krasteva, P.V., Baraquet, C., Harwood, C.S., Sondermann, H., and Navarro, M.V.A.S. (2016). Mechanistic insights into c-di-GMP-dependent control of the biofilm regulator FleQ from Pseudomonas aeruginosa. Proceedings of the National Academy of Sciences of the United States of America, 113: E209E218.

McDonald, M.J., Gehrig, S.M., Meintjes, P.L., Zhang, X.X., and Rainey, P.B. (2009). Adaptive divergence in experimental populations of Pseudomonas fluorescens. IV. Genetic constraints guide evolutionary trajectories in a parallel adaptive radiation. Genetics, 183: 1041-1053.

Mercado-Blanco, J., Rodrı́guez-Jurado, D., Hervás, A., and Jiménez-Dı́az, R.M. (2004). Suppression of Verticillium wilt in olive planting stocks by root-associated fluorescent Pseudomonas spp. Biological Control, 30: 474-486.

Molina-Henares, M.A., Ramos-González, M.I., Daddaoua, A., Fernández-Escamilla, A.M., and Espinosa-Urgel, M. (2017). FleQ of Pseudomonas putida KT2440 is a multimeric cyclic diguanylate binding protein that differentially regulates expression of biofilm matrix components. Research in Microbiology, 168: 36-45.

Montes-Osuna, N., Gómez-Lama Cabanás, C., Valverde-Corredor, A., Berendsen, R.L., Prieto, P., and MercadoBlanco, J. (2021). Assessing the involvement of selected phenotypes of Pseudomonas simiae PICF7 in olive root colonization and biological control of Verticillium dahliae. Plants, 10: 412.

Moxon, R., Bayliss, C., and Hood, D. (2006). Bacterial contingency loci: the role of simple sequence DNA repeats in bacterial adaptation. Annual Review of Genetics, 40: 307-333.

Nixon, B.T., Ronson, C.W., and Ausubel, F.M. (1986). Two-component regulatory systems responsive to environmental stimuli share strongly conserved domains with the nitrogen assimilation regulatory genes $n t r B$ and $n t r C$. Proceedings of the National Academy of Sciences of the United States of America, 83: $7850-7854$.

Orsi, R.H., Bowen, B.M., and Wiedmann, M. (2010). Homopolymeric tracts represent a general regulatory mechanism in prokaryotes. BMC Genomics, 11: 102.

Paul, R., Weiser, S., Amiot, N.C., Chan, C., Schirmer, T., Giese, B., and Jenal, U. (2004). Cell cycle-dependent dynamic localization of a bacterial response regulator with a novel di-guanylate cyclase output domain. Genes \& Development, 18: 715-727.

Rainey, P.B. (1999). Adaptation of Pseudomonas fluorescens to the plant rhizosphere. Environmental Microbiology, 1: 243-257.

Rainey, P.B., and Travisano, M. (1998). Adaptive radiation in a heterogeneous environment. Nature, 394: 69-72. Ramos-González, M.I., Travieso, M.L., Soriano, M.I., Matilla, M.A., Huertas-Rosales, Ó., Barrientos-Moreno, L., et al. (2016). Genetic dissection of the regulatory network associated with high c-di-GMP levels in Pseudomonas putida KT2440. Frontiers in Microbiology 7: 1093.

Rehm, B.H.A. (2010). Bacterial polymers: biosynthesis, modifications and applications. Nature Reviews Microbiology, 8: 578-592.

Reinders, A., Hee, C.-S., Ozaki, S., Mazur, A., Boehm, A., Schirmer, T., and Jenal, U. (2016). Expression and genetic activation of cyclic di-GMP-specific phosphodiesterases in Escherichia coli. Journal of Bacteriology, 198: 448-462. 
Remigi, P., Ferguson, G.C., McConnell, E., De Monte, S., Rogers, D.W., and Rainey, P.B. (2019). Ribosome provisioning activates a bistable switch coupled to fast exit from stationary phase. Molecular Biology and Evolution, 36: 1056-1070.

Richter, A.M., Possling, A., Malysheva, N., Yousef, K.P., Herbst, S., Kleist, M. von, and Hengge, R. (2020). Local c-di-GMP signaling in the control of synthesis of the E. coli biofilm exopolysaccharide pEtNcellulose. Journal of Molecular Biology, 432: 4576-4595.

Römling, U., Galperin, M.Y., and Gomelsky, M. (2013). Cyclic di-GMP: the first 25 years of a universal bacterial second messenger. Microbiology and Molecular Biology Reviews, 77: 1-52.

Ross, P., Weinhouse, H., Aloni, Y., Michaeli, D., Weinberger-Ohana, P., Mayer, R., et al. (1987). Regulation of cellulose synthesis in Acetobacter xylinum by cyclic diguanylic acid. Nature, 325: 279-281.

Ryjenkov, D.A., Simm, R., Römling, U., and Gomelsky, M. (2006). The PilZ domain is a receptor for the second messenger c-di-GMP. Journal of Biological Chemistry, 281: 30310-30314.

Schmidt, A.J., Ryjenkov, D.A., and Gomelsky, M. (2005). The ubiquitous protein domain EAL is a cyclic diguanylate-specific phosphodiesterase: enzymatically active and inactive EAL domains. Journal of Bacteriology, 187: 4774-4781.

Silby, M.W., Cerdeño-Tárraga, A.M., Vernikos, G.S., Giddens, S.R., Jackson, R.W., Preston, G.M., et al. (2009). Genomic and genetic analyses of diversity and plant interactions of Pseudomonas fluorescens. Genome Biology, 10: R51.

Spiers, A.J., Bohannon, J., Gehrig, S.M., and Rainey, P.B. (2003). Biofilm formation at the air-liquid interface by the Pseudomonas fluorescens SBW25 wrinkly spreader requires an acetylated form of cellulose. Molecular Microbiology, 50: 15-27.

Spiers, A.J., Kahn, S.G., Bohannon, J., Travisano, M., and Rainey, P.B. (2002) Adaptive divergence in experimental populations of Pseudomonas fluorescens. I. Genetic and phenotypic bases of wrinkly spreader fitness. Genetics, 161: 33-46.

Starkey, M., Hickman, J.H., Ma, L., Zhang, N., De Long, S., Hinz, A., et al. (2009). Pseudomonas aeruginosa rugose small-colony variants have adaptations that likely promote persistence in the cystic fibrosis lung. Journal of Bacteriology, 191: 3492-3503.

Steiner, S., Lori, C., Boehm, A., and Jenal, U. (2012). Allosteric activation of exopolysaccharide synthesis through cyclic di-GMP-stimulated protein-protein interaction. The EMBO Journal, 32: 354-368.

Stock, A.M., Robinson, V.L., and Goudreau, P.N. (2000). Two-component signal transduction. Annual Review of Biochemistry, 69: 183-215.

Stockwell, V.O., Davis, E.W., Carey, A., Shaffer, B.T., Mavrodi, D.V., Hassan, K.A., et al. (2013). pA506, a conjugative plasmid of the plant epiphyte Pseudomonas fluorescens A506. Applied Environmental Microbiology, 79: 5272-5282.

Stockwell, V.O., Johnson, K.B., Sugar, D., and Loper, J.E. (2010). Control of fire blight by Pseudomonas fluorescens A506 and Pantoea vagans C9-1 applied as single strains and mixed inocula. Phytopathology, 100: $1330-1339$.

Sudarsan, N., Lee, E.R., Weinberg, Z., Moy, R.H., Kim, J.N., Link, K.H., and Breaker, R.R. (2008). Riboswitches in eubacteria sense the second messenger cyclic di-GMP. Science, 321: 411-413. 
Tal, R., Wong, H.C., Calhoon, R., Gelfand, D., Fear, A.L., Volman, G., et al. (1998). Three $c d g$ operons control cellular turnover of cyclic di-GMP in Acetobacter xylinum: genetic organization and occurrence of conserved domains in isoenzymes. Journal of Bacteriology, 180: 4416-4425.

Tiwari, A., Ray, J.C.J., Narula, J., and Igoshin, O.A. (2011). Bistable responses in bacterial genetic networks: designs and dynamical consequences. Mathematical Biosciences, 231: 76-89.

Whitfield, G.B., Marmont, L.S., Bundalovic-Torma, C., Razvi, E., Roach, E.J., Khursigara, C.M., et al. (2020). Discovery and characterization of a Gram-positive Pel polysaccharide biosynthetic gene cluster. PLoS Pathogens, 16: e1008281.

Zhang, X.X., and Rainey, P.B. (2007). Genetic analysis of the histidine utilization (hut) genes in Pseudomonas fluorescens SBW25. Genetics, 176: 2165-2176.

Zordan, R.E., Galgoczy, D.J., and Johnson, A.D. (2006). Epigenetic properties of white-opaque switching in Candida albicans are based on a self-sustaining transcriptional feedback loop. Proceedings of the National Academy of Sciences of the United States of America, 103: 12807-12812.

\section{SUPPORTING INFORMATION}
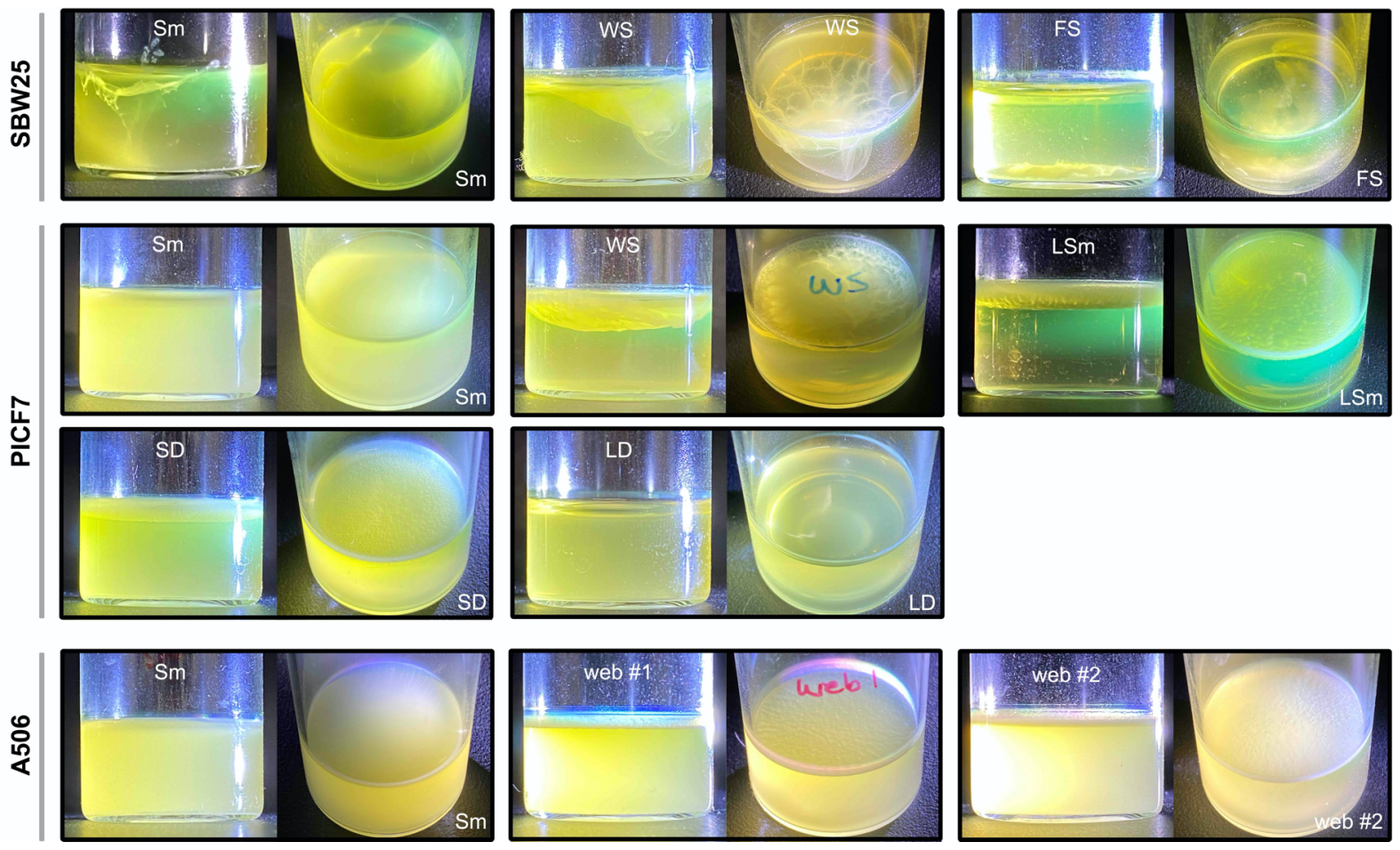

Figure S1. Five of the ten morphotypes isolated from 72-h microcosms have the emergent ability to form

mats at the air-liquid interface within 24-h. Ten classes of colony phenotypes were observed arising from 72$\mathrm{h}$ microcosms of SBW25, PICF7, and A506 (see Figure 2B). Of these, five classes (SBW25-WS, PICF7-WS, PICF7-LSm, PICF7-SD, and A506-Web) have the emergent ability to form mats within 24-h. The remaining five classes (SBW25-Sm, SBW25-FS, PICF7-Sm, PICF7-LD, and A506-Sm) do not form stable mats within 24 hours. All photographs taken at the same time point (24 h). Brightness of some images altered in Preview.

Table S1. Strains, plasmids, transposons, and oligonucleotides used in this study. 
Table S2. A comparison of regulatory and structural gene homologues in in $P$. fluorescens SBW25, $P$. simiae PICF7, and P. fluorescens A506.

Table S3. Mutations predicted by whole genome re-sequencing of ten SBW25-WS, ten PICF7-WS, and ten A506-Web isolates. Illumina whole genome re-sequencing was performed for each isolate. This file contains (i) a summary of the raw read numbers and coverage information ("summary" tab), and (ii) a list of mutations predicted by breseq (Deatherage and Barrick, 2014), for each isolate (separated into "sbw25", "picf7", and "a506" tabs). WS mutations identified in PICF7-WS and SBW25-WS are further described in Text $\mathrm{S} 1$, and the possible duplications in A506 isolates (including wild type) are further described in Text S2.

Table S4. Suppressor analysis of the PICF7-WS phenotype. Genotype and phenotype details of 100 nonwrinkly transposon mutants from four PICF7-WS backgrounds (PICF7-WS3, WS4, WS7, and WS8; carrying mutations in $w s p F, a w s X, m w s R$, and $w s p F$, respectively). Mutants were obtained by screening approximately $\sim 15,600$ transposon mutants from eleven independent conjugations for loss of the WS phenotype conferred by the WS mutation; each mutant forms smooth colonies, and cannot form a static microcosm mat within 24 hours. Mutants are classified according to the cellular function likely to be affected by the insertion. The precise genomic location of the transposon is indicated as the first base on the genomic forward strand downstream of the $3^{\prime}$ transposon terminus. For mutants of particular interest, a Cre-deletion (removing most of the transposon and thus eliminating polar effects) was obtained and analysed. Green highlighting shows transposon insertions in the pel biosynthetic locus; grey highlighting shows transposon insertions in loci involved in the regulation of Pel production (wsp, aws, or $m w s R$, depending on the WS mutation carried by the genotype being mutagenized).

Text S1. Details of WS mutations in PICF7-WS and SBW25-WS.

Text S2. Analysis of the high coverage regions in P. fluorescens A506 isolates.

Text S3. Extended methods for the construction of scar-free mutations in P. fluorescens A506. 